\title{
Critical temperature in QCD with two flavors of dynamical quarks*
}

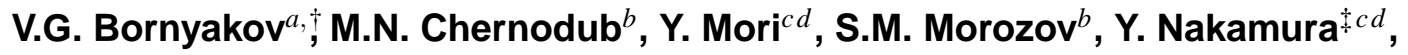 \\ M.I. Polikarpov ${ }^{b}$, G. Schierholz ${ }^{e}$, A.A. Slavnov ${ }^{f, \S}$ H. Stüben ${ }^{g}$, T. Suzuki ${ }^{c} d, \pi$ \\ ${ }^{a}$ Institute for High Energy Physics, RU-142284 Protvino, Russia \\ ${ }^{b}$ ITEP, B.Cheremushkinskaya 25, RU-117259 Moscow, Russia \\ ${ }^{c}$ Institute for Theoretical Physics, Kanazawa University, Kanazawa 920-1192, Japan \\ ${ }^{d}$ RIKEN, Radiation Laboratory, Wako 351-0158, Japan \\ ${ }^{e}$ NIC/DESY Zeuthen, Platanenallee 6, D-15738 Zeuthen, Germany \\ ${ }^{f}$ Steklov Mathematical Institute, Vavilova 42, RU-117333 Moscow, Russia \\ ${ }^{g}$ Konrad-Zuse-Zentrum für Informationstechnik Berlin, D-14195 Berlin, Germany \\ E-mail: yoshi@hep.s.kanazawa-u.ac.jp
}

\begin{abstract}
We present results obtained in QCD with two flavors of non-perturbatively improved Wilson fermions at finite temperature on $16^{3} \times 8$ and $24^{3} \times 10$ lattices. We determine the transition temperature in the range of quark masses $0.6<m_{\pi} / m_{\rho}<0.8$ at lattice spacing $\mathrm{a} \approx 0.1 \mathrm{fm}$ and extrapolate the transition temperature to the continuum and to the chiral limits. We also discuss the order of phase transition.
\end{abstract}

XXIIIrd International Symposium on Lattice Field Theory

25-30 July 2005

Trinity College, Dublin, Ireland

*This work is supported by the SR8000 Supercomputer Project of High Energy Accelerator Research Organization (KEK). A part of numerical measurements has been done using NEC SX-5 at Research Center for Nuclear Physics (RCNP) of Osaka University. The numerical simulations of this work were done using RSCC computer clusters in RIKEN. We wish to acknowledge the support of the computer center at RIKEN.

${ }^{\dagger}$ VGB, MNC and MIP are supported by grants 04-02-16079, RFBR-DFG-03-02-04016, DFG-RFBR 436 RUS 113/739/0, CRDF award RPI-2364-MO-02 and MK-4019.2004.2.

† Speaker.

$\S$ A.A.S. is supported by grant Scientific School grant 2052-2003.1.

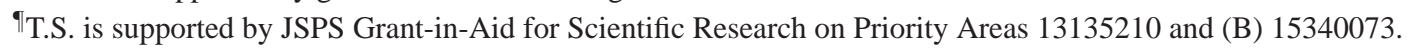




\section{Introduction}

Knowledge of the QCD phase structure is important in order to understand the physics of the early Universe, structure of neutron stars, processes in heavy ion collisions, etc. In order to obtain predictions for the real world from lattice QCD, we have to extrapolate the lattice data to the continuum and to the chiral limit. Determination of the transition temperature $T_{c}$ was studied by many groups. $T_{c}$ from staggered fermions with 2 and $2+1$ flavors of dynamical quarks [1, 2, 3, 4], and from Wilson fermions with 2 flavors [5] was found to be consistent in the chiral limit. Edwards and Heller [6] determined $T_{c}$ for $L_{t}=4$ and 6 using nonperturbatively improved Wilson fermions. We compute $T_{c}$ on finer lattices with $L_{t}=8$ and 10 and extrapolate our high statistics data to the continuum limit. Our results for $L_{t}=8$ at $\beta=5.2$ were previously reported in Ref. [7].

\section{Simulation}

We use the Wilson gauge action and the non-perturbatively improved Wilson fermions with $c_{s w}$ given in Ref. [8]. Configurations are generated on $16^{3} \times 8\left(\beta=5.2\right.$ and 5.25) and $24^{3} \times 10$ ( $\beta=5.2$ ) lattices at various $\kappa$. The values of $\kappa$ and the corresponding number of configurations for $24^{3} \times 10$ and $16^{3} \times 8$ lattices can be found in Table 1 . We use $\mathrm{T}=0$ results obtained by QCDSFUKQCD to fix the scale. The contour plot of lines of constant $r_{0} / a$ and $m_{\pi} / m_{\rho}$ is shown in Fig. 1 .

\begin{tabular}{|l|r||l||l||l|r|}
\hline \multicolumn{2}{|c||}{$24^{3} \times 10$} & \multicolumn{4}{c|}{$16^{3} \times 8$} \\
\hline \multicolumn{2}{|c||}{$\beta=5.2$} & \multicolumn{2}{c|}{$\beta=5.2$} & \multicolumn{2}{c|}{$\beta=5.25$} \\
\hline$\kappa$ & \# of conf. & $\kappa$ & \# of traj. & $\kappa$ & \# of traj. \\
\hline 0.1348 & 678 & 0.1330 & 7,120 & 0.1330 & 1,540 \\
\hline 0.1352 & 2058 & 0.1335 & 4,500 & 0.1335 & 7,439 \\
\hline 0.1353 & 2725 & 0.1340 & 3,000 & 0.13375 & 9,225 \\
\hline 0.1354 & 3934 & 0.1343 & 6,615 & 0.1339 & 12,470 \\
\hline 0.1355 & 3816 & 0.1344 & 11,275 & 0.1340 & 19,479 \\
\hline 0.1356 & 1730 & 0.1345 & 9,077 & 0.1341 & 13,750 \\
\hline 0.1358 & 1210 & 0.1348 & 5,813 & 0.13425 & 5,155 \\
\hline 0.1360 & 617 & 0.1355 & 5,650 & 0.1345 & 2,650 \\
\hline & & 0.1360 & 3,699 & 0.1350 & 1,780 \\
\hline
\end{tabular}

Table 1: Simulation statistics.

\section{Critical temperature}

We use the Polyakov loop susceptibility to determine the transition point. We identify the critical value of $\kappa, \kappa_{t}$, as a point, where the Polyakov loop susceptibility reaches its maximum. Applying a Gaussian fit in the vicinity of the maximum (as shown in Figs. 2, 3 and 4) we find $\kappa_{t}=$ $0.13542(6)$ at $\beta=5.2$ on $24^{3} \times 10, \kappa_{t}=0.13444(6)$ at $\beta=5.2$ on $16^{3} \times 8$, and $\kappa_{t}=0.13406(6)$ at $\beta=5.25$ on $16^{3} \times 8$. Using the Sommer scale $r_{0}=0.5 \mathrm{fm}$ and interpolating $r_{0} / a$ to the critical point, 


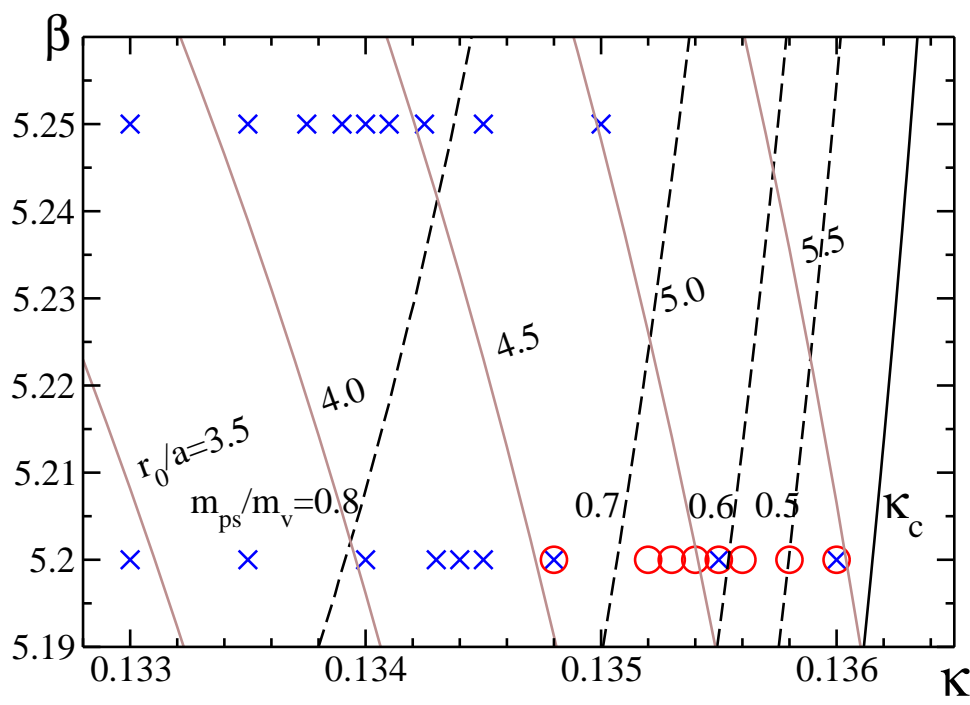

Figure 1: Lines of constant $r_{0} / a$ (solid lines) and constant $m_{\pi} / m_{\rho}$ (dotted lines) at $T=0$. Crosses and circles indicate points where simulations are done.

we obtain for the critical temperatures $T_{c}=197(2) \mathrm{MeV}\left(\right.$ at $\left.m_{\pi} / m_{\rho}=0.63(2)\right), T_{c}=212(2) \mathrm{MeV}$ (at $m_{\pi} / m_{\rho}=0.76(1)$ ), and $T_{c}=217(2) \mathrm{MeV}\left(\right.$ at $\left.m_{\pi} / m_{\rho}=0.81(1)\right)$, respectively.
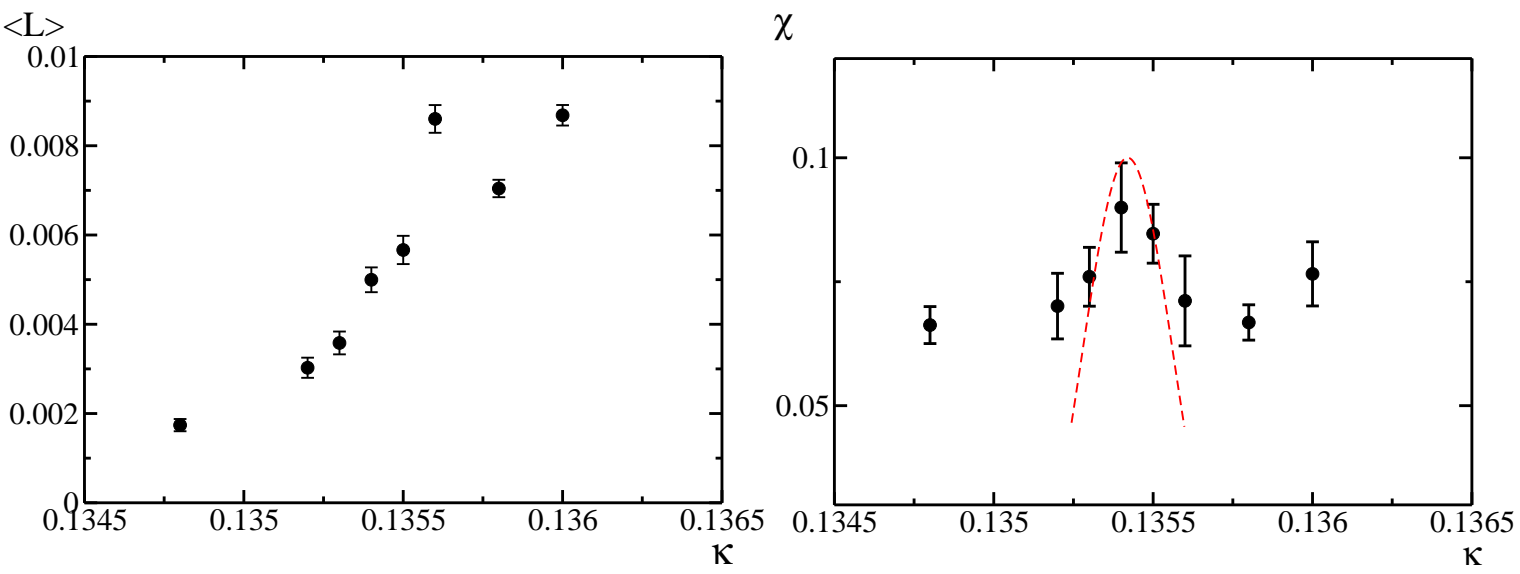

Figure 2: Polyakov loop (left) and its susceptibility (right) at $\beta=5.2$ on $24^{3} \times 10$ lattice.

In Fig. 5 we compare our result for the critical temperature with those of Refs. [1], [5] and [6], where we have assumed $\sqrt{\sigma}=425 \mathrm{MeV}$, and $\mathrm{m}_{\mathrm{v}}$ and $\mathrm{m}_{\mathrm{ps}}$ are vector meson mass and pseudoscalar mass obtained on the zero-temperature lattice. Our result is in quantitative agreement with the results of the Bielefeld group and CP-PACS. This is reassuring, as [1], [5] and [6] work at larger lattice spacing.

\section{Continuum and chiral limits}

In order to estimate $T_{c}$ in the continuum and the chiral limits we apply the extrapolation for- 

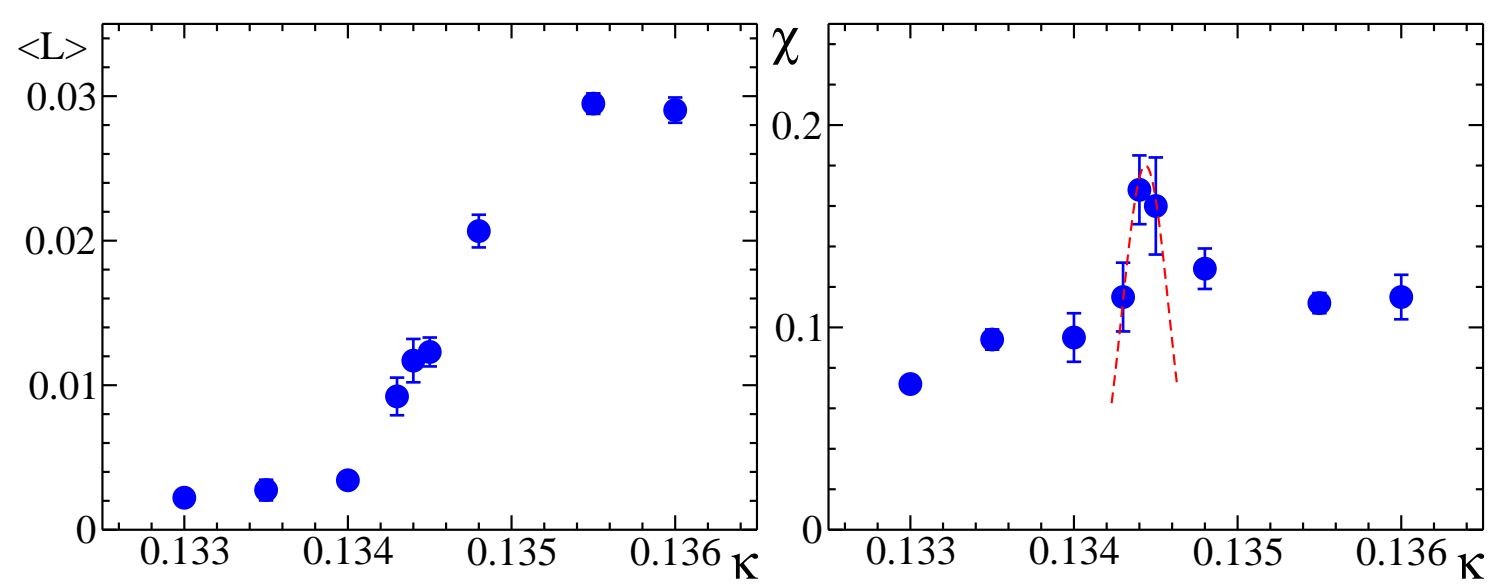

Figure 3: Polyakov loop (left) and its susceptibility (right) at $\beta=5.2$ on $16^{3} \times 8$ lattice.
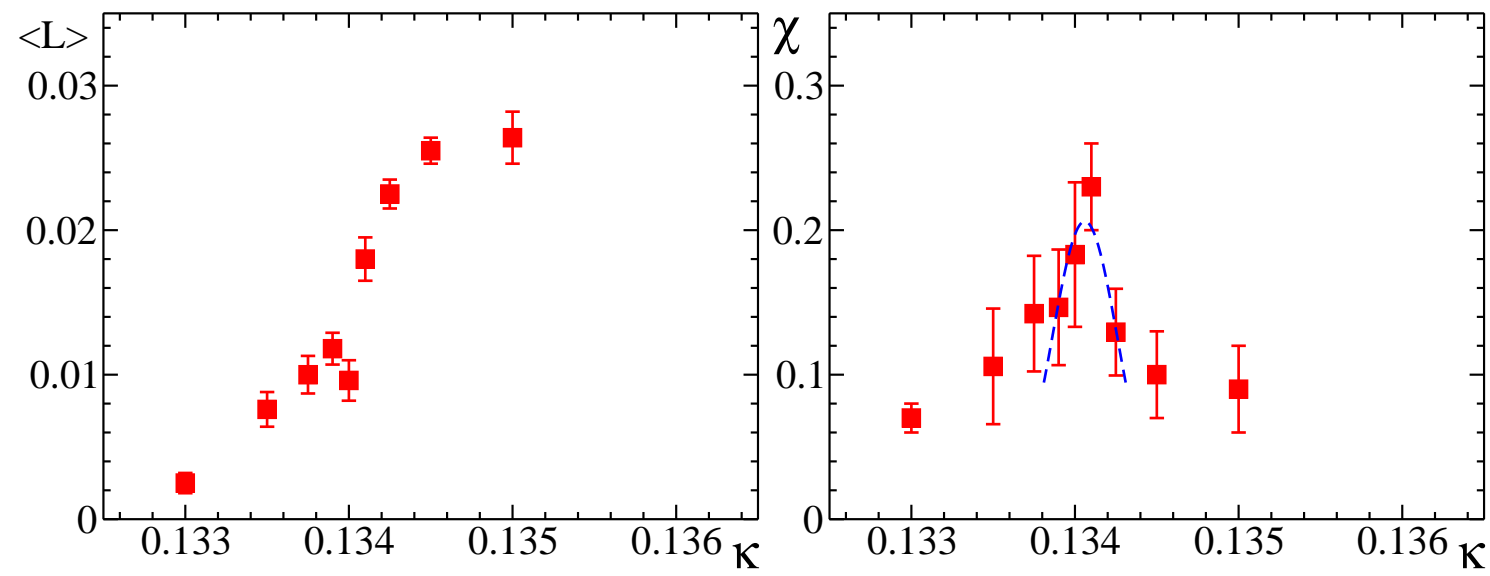

Figure 4: Polyakov loop (left) and its susceptibility (right) at $\beta=5.25$ on $16^{3} \times 8$ lattice.
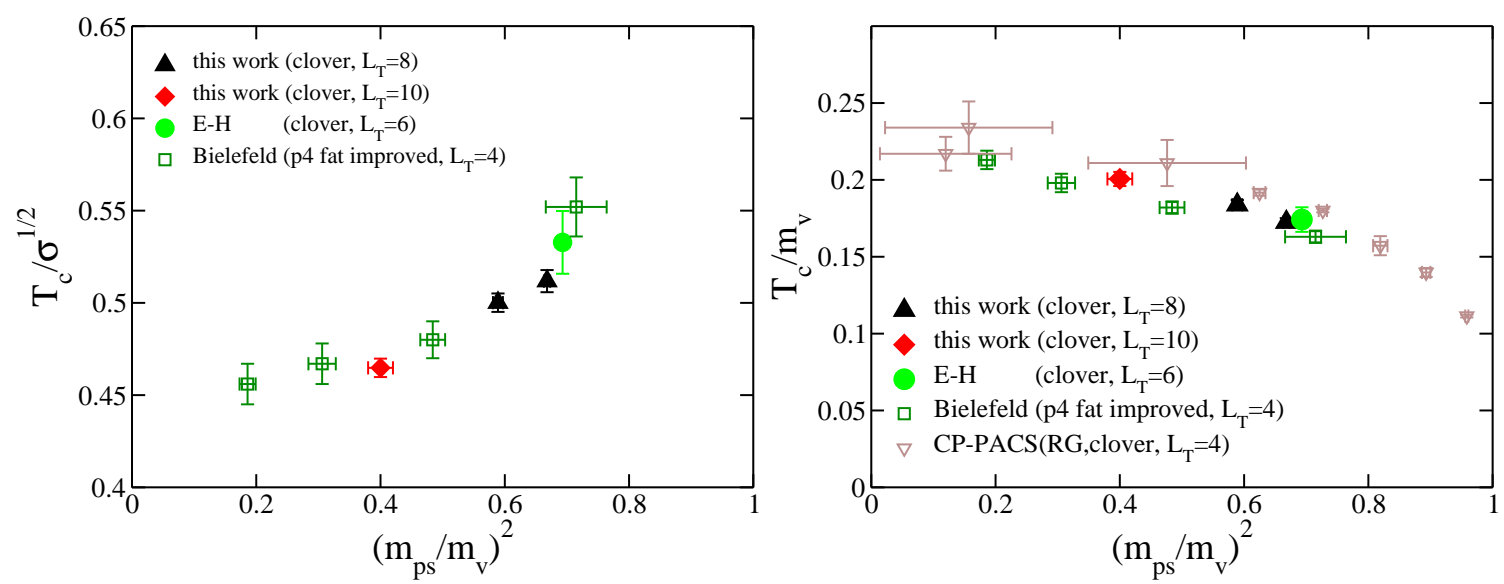

Figure 5: (left) The transition temperature $T_{c}$ in units of the string tension $\sigma(T=0)$ as a function of $m_{\mathrm{ps}} / m_{\mathrm{v}}$ and (right) $T_{c}$ in units of $m_{\mathrm{v}}$ as a function of $\left(m_{\mathrm{ps}} / m_{\mathrm{v}}\right)^{2}$ (right). The data is from this work (the up triangles and the diamond), Ref. [1] (the squares), Ref. [6] (the circle), and Ref. [5] (the down triangles). 
mula, which takes into account the lowest order corrections coming from the finite lattice spacing and quark masses. We use two extrapolation functions: the first one is of the bare quark mass type (TYPE 1) and the second one is of the pseudoscalar mass type (TYPE 2).

$$
\begin{array}{ll}
\text { TYPE } 1: & T_{c} r_{0}=T_{c}^{m_{q}, a \rightarrow 0} r_{0}+C_{a}\left(\frac{a}{r_{0}}\right)^{2}+C_{q}\left(\frac{1}{\kappa}-\frac{1}{\kappa_{c}}\right)^{\frac{1}{\beta \delta}}, \\
\text { TYPE 2: } & T_{c} r_{0}=\left(T_{c} r_{0}\right)^{m_{q}, a \rightarrow 0}+C_{a}\left(\frac{a}{r_{0}}\right)^{2}+C_{q}\left(m_{p s}\right)^{\frac{2}{\beta \delta}} .
\end{array}
$$

where $T_{c}^{m_{q}, a \rightarrow 0}$ corresponds to the extrapolated value of the critical temperature, and $\beta$ and $\delta$ are the critical indices. Since $O(a)$ improved Wilson fermions are employed in this studies, we expect corrections of $O\left(a^{2}\right)$ only.

We make an attempt to fit four values for $T_{c} r_{0}$ (see Table 2), obtained at rather large quark masses, in order to estimate the extrapolation parameters used in Eqs. (4.14.2). If the transition in two-flavor QCD is of the second order, then the transition is expected to belong to the universality class of the $3 D O(4)$ spin model with $1 / \beta \delta \approx 0.54$. Current lattice calculations suggest that transition in QCD for physical quark masses is not a true phase transition but a crossover [1, 2, 3, 4, 5, 10]. Since results obtained by CP-PACS indicated a good $O(4)$ scaling [5], we extrapolate the value of the critical temperature using the value of 0.54 as $1 / \beta \delta$. We rely on the assumption that the finite temperature phase transition in two-flavor QCD is second order. The best fit parameters are presented in Table 3.

\begin{tabular}{|c|c|c|c|c|c|}
\hline $\mathrm{T}_{c} \mathrm{r}_{0}$ & $\mathrm{a} / \mathrm{r}_{0}$ & $1 / \kappa-1 / \kappa_{\mathrm{c}}$ & $\mathrm{m}_{\mathrm{ps}}$ & $\mathrm{L}_{\mathrm{t}}$ & $\beta$ \\
\hline $0.501(5)$ & $0.200(2)$ & $0.040(4)$ & $1.58(6)$ & $10(\mathrm{DIK})$ & 5.2 \\
\hline $0.539(5)$ & $0.232(3)$ & $0.094(4)$ & $2.25(4)$ & $8(\mathrm{DIK})$ & 5.2 \\
\hline $0.551(5)$ & $0.227(2)$ & $0.123(4)$ & $2.61(4)$ & $8(\mathrm{DIK})$ & 5.25 \\
\hline $0.57(2)$ & $0.290(9)$ & $0.174(12)$ & $2.74(5)$ & $6(\mathrm{E}-\mathrm{H})$ & 5.2 \\
\hline
\end{tabular}

Table 2: The available data for the critical temperature $T_{c} r_{0}$.

\begin{tabular}{|c|c|c|c|c|c|}
\hline $1 / \beta \delta$ & $\left(\mathrm{T}_{c} \mathrm{r}_{0}\right)^{\mathrm{m}_{\mathrm{q}}, \mathrm{a} \rightarrow 0}$ & $\mathrm{C}_{\mathrm{a}}$ & $\mathrm{C}_{\mathrm{q}}$ & $\chi^{2} /$ dof & TYPE \\
\hline 0.54 & $0.439(7)$ & $0.1(2)$ & $0.33(3)$ & 0.09 & 1 \\
\hline 0.54 & $0.420(7)$ & $0.5(1)$ & $0.036(2)$ & 0.04 & 2 \\
\hline
\end{tabular}

Table 3: The best fit parameters for the case if the transition is of second order.

We get the critical temperature in the continuum and in the chiral limits,

TYPE1 fitting: $\quad T_{c}=173(3) \mathrm{MeV}$,

TYPE2 fitting: $\quad T_{c}=166(3) \mathrm{MeV}$.

The value of $T_{c}$ extrapolated by using the TYPE 1 fitting agrees with the values obtained by other groups, but the value from the TYPE 2 fitting is slightly smaller. Both fits should give the same result. The difference gives an indication of the systematic error.

Finally, we mention the results of Ref. [9] which support the first order of the transition. If the transition is indeed of the first order, then $1 / \beta \delta=1$. In order to test the sensitivity of $T_{c}$ to the order of the transition, we also performed a fit with $1 / \beta \delta=1$. 


\begin{tabular}{|c|c|c|c|c|c|}
\hline $1 / \beta \delta$ & $\left(\mathrm{T}_{\mathrm{c}} \mathrm{r}_{0}\right)^{\mathrm{m}_{\mathrm{q}}, \mathrm{a} \rightarrow 0}$ & $\mathrm{C}_{\mathrm{a}}$ & $\mathrm{C}_{\mathrm{q}}$ & $\chi^{2} / \mathrm{dof}$ & $\mathrm{TYPE}$ \\
\hline 1 & $0.486(17)$ & $0.0(4)$ & $0.5(1)$ & 0.11 & 1 \\
\hline 1 & $0.460(11)$ & $0.5(2)$ & $0.09(2)$ & 0.08 & 2 \\
\hline
\end{tabular}

Table 4: The best fit parameters for the case if the transition is of first order.

$$
\text { TYPE1 fitting: } \quad T_{c}=192(7) \mathrm{MeV}, \quad \text { TYPE2 fitting: } \quad T_{c}=182(4) \mathrm{MeV} .
$$

These values are rather large compared to the value obtained from $N_{f}=2+1$ staggered fermions in Ref. [4]. The results of our fits do not discriminate between the first and the second order phase transitions because of rather large errors in the $T_{c} r_{0}$ values. We are continuing simulations on $24^{3} \times 10$ lattice in order to get a better precision of the $T_{c}$ value for this lattice.

\section{References}

[1] F. Karsch, E. Laermann and A. Peikert, Quark mass and flavor dependence of the QCD phase transition, Nucl. Phys. B 605 (2001) 579 [hep-lat/ 0012023 ].

[2] Z. Fodor and S. D. Katz, Critical point of $Q C D$ at finite $T$ and $m u$, lattice results for physical quark masses, JHEP 0404, 050 (2004) [hep-lat/ 0402006 ].

[3] P. Petreczky, The finite temperature transition in $(2+1)$-flavour QCD with staggered HYP action, $J$. Phys. G 30 (2004) S1259.

[4] C. Bernard et al. [MILC Collaboration], QCD thermodynamics with three flavors of improved staggered quarks, Phys. Rev. D 71 (2005) 034504 [hep-lat/ 0405029 ].

[5] A. Ali Khan et al. [CP-PACS Collaboration], Phase structure and critical temperature of two flavor $Q C D$ with renormalization group improved gauge action and clover improved Wilson quark action, Phys. Rev. D 63 (2001) 034502 [hep-lat/ 0008011$].$

[6] R. G. Edwards and U. M. Heller, Thermodynamics with dynamical clover fermions, Phys. Lett. B 462, 132 (1999) [hep-lat/9905008].

[7] V. G. Bornyakov et al. [DIK Collaboration], Finite temperature QCD with two flavors of non-perturbatively improved Wilson fermions, Phys. Rev. D 71, 114504 (2005)

[hep-lat/0401014].

[8] K. Jansen and R. Sommer [ALPHA collaboration], $O(\alpha)$ improvement of lattice $Q C D$ with two flavors of Wilson quarks, Nucl. Phys. B 530, 185 (1998) [Erratum-ibid. B 643, 517 (2002)] [hep-lat/9803017].

[9] M. D'Elia, A. Di Giacomo and C. Pica, The order of the chiral transition in $N(f)=2 Q C D, N u c l$. Phys. B (Proc. Suppl.) 140 (2005) 541 [hep-lat/ 0408008 ].

[10] F. Karsch et al., Where is the chiral critical point in 3-flavor QCD?, Nucl. Phys. Proc. Suppl. 129 (2004) 614 [hep-lat/0309116]. 